\title{
sEMG-based Hand Gesture Classification with Transient Signal
}

\author{
Yue Zhang ${ }^{1}$, Jiahui $\mathrm{Yu}^{2}$ Dalin $\mathrm{Zhou}^{2}$, and Honghai $\mathrm{Liu}^{2}$ \\ 1 College of Computer Science and Technology, Zhejiang University of Technology, \\ Hangzhou, China PR, \\ zhangyuemessi@163.com \\ 2 School of Computing, University of Portsmouth, Portsmouth, United Kingdom \\ jiahui.yu@port.ac.uk, dalin.zhou@port.ac.uk, honghai.liu@port.ac.uk
}

\begin{abstract}
Surface electromyography (sEMG) can provide a novel control method for human machine interface (HMI) with the improvement of signal decoding technology. sEMG-based hand gesture recognition is the key part of HMI control strategy. However, unstable and complex daily used scenarios hinder the further development of sEMG-based control strategy. In this paper, we concentrate on the data preprocessing part. Three different signal segments were extracted including the transient signal segments between gestures, standard signal segments and stationary signal segments which is smaller than standard segment. By setting up several experiments to analyze and evaluate the classification performance with these transient information. Our research found that transient signal segments can reflects more effective information than the stationary signals in inter-subject scenes. It gained more classification accuracy and stability. In addition, it also performance better in other two scenes in ten hand gesture recognition in intra-session and inter-session.
\end{abstract}

Keywords: sEMG, Feature extraction, traditional classifier, transient signal

\section{Introduction}

Surface electromyography is a bioelectrical signal that represents the impulses of motor units during skeletal muscle fiber excitement and contraction. It is a comprehensive temporal and spatial characteristic map of electrical activity of complex subepidermal muscles, which can be extracted from the surface of the skin by recorded electrodes. There are different degrees of correlation between sEMG signals and muscle activity and function, which reflects neuromuscular activity to a certain extent(see [1]).

Given the characteristic that sEMG signals are easy to collect, non-invasive, and bionic, many researchers have devoted themselves to studying the essential characteristics of sEMG signals, revealing the physiological mechanism that they generate and the muscle motor systems they reflect. At present, sEMG is widely used in the diagnosis and rehabilitation of neuromuscular diseases including muscle physiology, muscle metabolism, rehabilitation medicine, sports and 
other fields(see [2]). In addition, sEMG can also be used to provide a safe, nonintrusive control method for advanced prosthesis control, functional electrical stimulation and other advanced HMI(see [3] and [4]). The sEMG-based gesture recognition studied in this paper is a key part of the control of multifunctional dexterous prosthetic hand. Also, it is an important research direction in the field of rehabilitation and intelligent control.

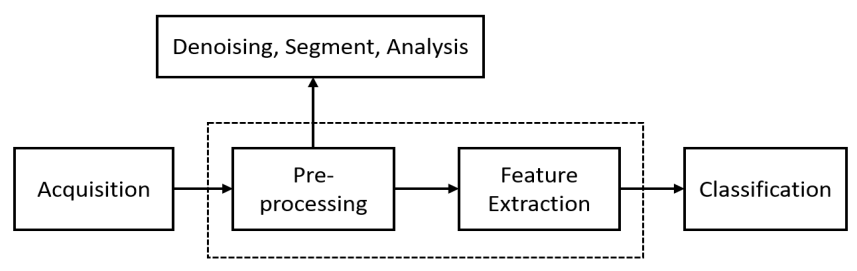

Fig. 1. Signal processing flow

Currently, gesture recognition based on pattern recognition has been regarded as the optimizing approach among different kind of strategies(see [5]). A general pattern recognition system can be divided into four parts: data collection, data preprocessing, feature extraction and classification, as shown in figure 1. Each part has a significant impact on the results of gesture recognition. In data collection, we always use an advanced commercial equipment to extract weak effective signals. Data preprocessing is always combined with the part of feature extraction(see [6]), it plays a vital role in feature extraction. The raw signal from acquisition device is often accompanied by some unstable noise which hinder the classification performance. Data preprocessing can remove the noise by some software filtering methods, such as high-pass filtering or low-pass filtering. In addition, this part can also extract and segment the needed part of signal. Feature extraction plays a critical role to the classification performance(see [7] and [8] and [9]). It obtains the characteristic parameters of each analysis window to characterize the characteristic vector of the EMG signal. So far, a large number of sEMG features have been investigated for hand gesture classification, and they can be summarized into four types: time domain (TD), frequency domain (FD), time-frequency domain (TFD) and parametric model analysis. Phinyomark et al.(see [10]) compared thirty-seven TD features and FD features, the experimental result demonstrated that the top three sEMG features could reach the accuracy more than $80 \%$. Besides that, it reveals that most of the TD features were redundant and better than the FD features. Classification is used to build a model which can map the characteristic vector to different motions. Generally, some classic supervised classification methods are used in gesture recognition, such as linear discriminant analysis (LDA), support vector machine (SVM), Naive Bayes and K nearest neighbor (KNN). 
Although myoelectric pattern recognition approach can achieve good performance in special laboratory environment, but failed in daily life due to the changes between training data and testing data generated by muscle fatigue(see [7]), electrode shifts(see [8]), and arm position changes(see [9]). To overcome the influence of these external factors, some methods were proposed. Deep learning (DL) has become popular in recent years and has achieved outstanding performance in many fields, such as image processing, speech recognition and natural language processing(see [11]). Convolutional neural network (CNN) and recurrent neural network (RNN), two classic network architectures of deep learning, have been applied to the gesture classification of sEMG. Manfredo et al.(see [12]) use a simple CNN architecture which is combined by four convolutional layers, one fully connected layer and a softmax function to classify the gestures on DB1 in Ninapro database. Finally, it obtains $4.53 \%$ higher accuracy than the classical classification methods. Geng et al.(see [13]) applied a deep CNN to a high-density EMG, and achieved an accuracy of $89.3 \%$ with a single frame and $99.0 \%$ with over 40 frames for 8 gestures recognition. Adaptive learning can enhance the robust of algorithm by adjusting the parameters of training model when external factors changes. Liu J.(see [14]) proposed an adaptive unsupervised classifier based on support vector machine and incremental learning method. This classifier considers the real-time changes between testing data and training data when predict the classification label. Then some adjustments on these difference would be added to the classification model in an unsupervised manner. Thus, continuously updating the model parameters makes the classifier adaptive to the changes. Chen et al.(see [15]) extend two off-line pattern recognition methods, linear discriminant analysis and quadratic discriminant analysis, to self-enhancing methods which continuously updating the class mean vectors, the class covariance and the pooled covariance using the testing data respectively.

In general, researchers always place their focus point on feature extraction and classification. They regard the signal of stationary section of a gesture as the most representative data. However, when capturing sEMG data of a repeated gesture in different session, the experimental volunteer completes the motion with different force because of fatigue or force information missing. Thus, the different session of stationary section exists some unstable factors. In this paper, we will extract transient signal segments when a hand gesture changes as the input data. Several TD features and supervised classifications will be used to compare with traditional processes.

\section{Material and methods}

\section{$2.1 \quad$ Subjects}

Eight able-bodied subjects are employed in to investigate the classification performance with the transient signal segments of sEMG signals. ( 1 female, 7 males, age: $25 \pm 5$,height: $175 \pm 10 \mathrm{~cm}$, weight: $65 \pm 10 \mathrm{~kg})$. Subjects have no previous history of neuropathies or traumas to the upper limbs and fingers. They were obtained full informed consent according to the Declaration of Helsinki. All subjects 
receive the relevant guidelines before capturing sEMG data during the entire experiment. The data capture cycle was one week.

\subsection{Apparatus}

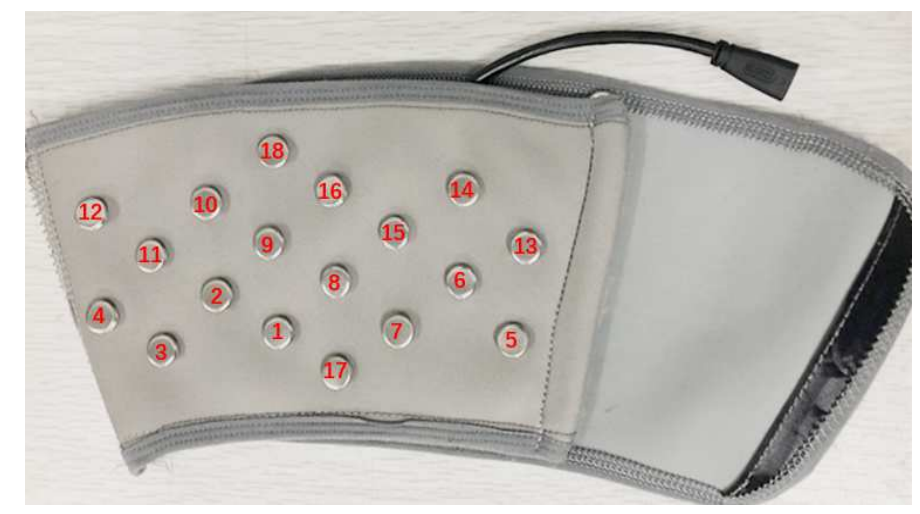

Fig. 2. The electrode sleeve for sEMG signal recording, 16 monopolar channels record the differential voltage between electrode 1-16 and electrode 17 and 18 provides the bias voltage to remove DC offset.

To capture the sEMG signal on the forearm, a multi-channel sEMG acquisition system, developed by our research group(see [16]), was employed to record the multi-channel sEMG signals. This device supports up to 16 bipolar EMG channels, 5000 amplification gains, $1 \mathrm{kHz}$ sampling frequency and 12 bits ADC resolution. To remove the motion artefacts, a band-pass filter with cutoff frequencies at $20 \mathrm{~Hz}$ and $500 \mathrm{~Hz}$ is integrated in the device. In addition, a notch filter with the fundamental frequency at $50 \mathrm{~Hz}$ is also deployed in hardware to remove the power frequency signal from the power line. The noise of each EMG channel was less than $1 \mathrm{~V}$. The entire device was powered by a $3.3 \mathrm{~V}$ rechargeable lithium battery, supporting continuous signal recording for up to ten hours.

This acquisition system use an electrode sleeve as signal conducting and recording equipment, as seen fig. 2, which embedded 18 electrodes in Zig configuration on an elastic fabric sleeve(see [17]). The diameter of each electrode is 12 $\mathrm{mm}, 25 \mathrm{~mm}$ vertical and $30 \mathrm{~mm}$ vertical and horizontal distances between two electrodes. 1 to 16 represent 16 independent channels, one reference electrode marked as 17 and one bias electrode marked as 18. All 16 channels are recorded synchronously.

\subsection{Acquisition Protocol}

Before the experiment, each subject should wear the electrode sleeve in the right way. They were required to be familiar with the experimental process and 


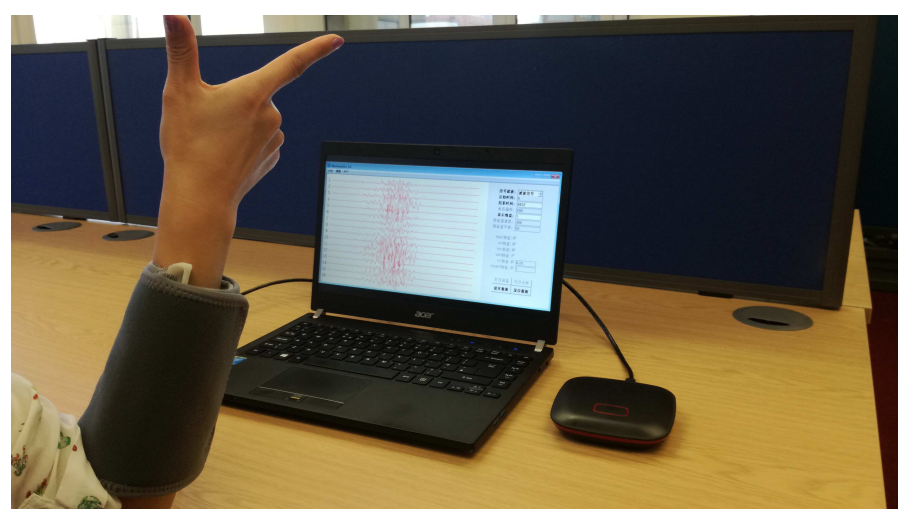

Fig. 3. The scenes for sEMG data recording.

practice the pre-defined hand gestures for several minutes. The selected of hand gestures in this experiment refer to the NinaPro dataset(see [18]) and CSLHDEMG database(see [19]) which are the two popular sEMG databases. All the hand gestures can be seen in fig. 4. Among them, the first row contains five finger-related gestures, they are (1) tip pinch, (2) flexion of ring and little finger, thumb flexed over ring and little finger, (3) flexion of middle, (4) ring and little finger, middle and ring flexion, and (5) thump up. The second row is wristrelated gestures, includes (1) hand close, (2) hand open, (3) wrist deviation, (4) wrist extension and (5) wrist flexion. Each subject was asked to complete the action with norm strength.

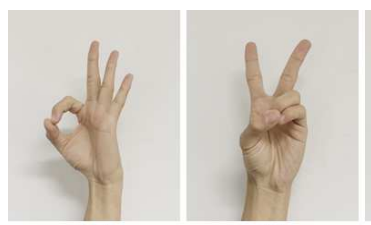

(1)

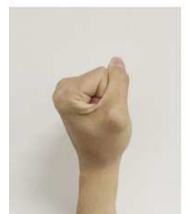

(6)
(2)

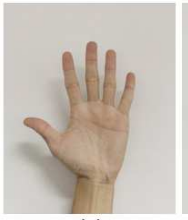

(7)

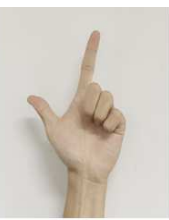

(3)

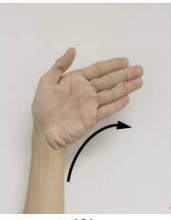

(8)

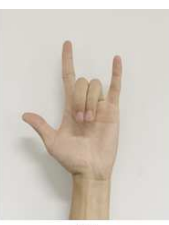

(4)

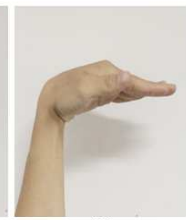

(9)

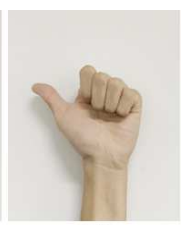

(5)

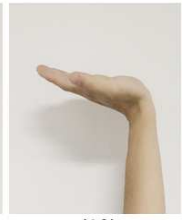

(10)

Fig. 4. Ten gestures were used for this experiment. (1)tip pinch, (2)flexion of ring and little finger, thumb flexed over ring and little finger, (3)flexion of middle, (4)ring and little finger, middle and ring flexion, and (5)thump up, (6)hand close (hc), (7)hand open (ho), (8)wrist radial deviation (wrd), (9)wrist extension (we), (10)wrist flexion (wf). 
Throughout the acquisition process, each subject wore the electrode sleeve on the left forearm and keep a standard sitting position in comfortable on the chair, as seen in fig. 2. Ten hand gestures were performed in sequence and each gesture maintained for at least $12 \mathrm{~s}$. In order to avoid muscle fatigue, subjects were asked to relax their hand for at least 10s between two adjacent gestures in a session. After finishing the ten hand gestures collected in short period, the subjects were asked to have a rest for half an hour. In this time period, subject can move around the office without removing the sleeve. The second session and third session were carried out in the same way. So, we separately gained three sessions from each subject and all sessions have the same electrode distribution. After three days, another three sessions were carried out to collect sEMG signals from each subject in the same way. It was worth pointing out that no predefined contraction force or elbow angle were applied in the experiment to mimic the real application of a sEMG based HMI as much as possible, although it was well known that muscular contract force and arm position could influence the robustness of patter recognition system (see [20]).

\subsection{Data Preprocessing}

Table 1. The format of EMG database.

\begin{tabular}{ccc} 
& \multicolumn{3}{c}{ aaa-ccc.mat } \\
\hline Name & Type & Description \\
\hline subject & scalar & The subject ID \\
group & scalar & Gesture group ID \\
data & $110000 \times 16$ matrix Raw sEMG signals of five gestures \\
\hline
\end{tabular}

\begin{tabular}{ccc} 
& \multicolumn{2}{c}{ ss-aaa-bbb-ccc.mat } \\
\hline Name & Type & Description \\
\hline shape & scalar & The segmented shape ID \\
subject & scalar & The subject ID \\
gesture & scalar & The gesture ID \\
trial & scalar & The trail ID \\
data & $10000 \times 16$ matrix & sEMG data for standard \\
& $4000 \times 16$ matrix & sEMG data for one transient \\
& $6000 \times 16$ matrix & sEMG data for one stationary \\
\hline
\end{tabular}

According to the acquisition protocol, each session contains ten hand gestures and each gesture maintained for $12 \mathrm{~s}$. To evaluate the performance of transient segments of sEMG signals in classification, we segment the raw data into three 
shapes: standard signal segments, transient signal segments, stationary signal segments. First, we extract standard section part. Only the later $10 \mathrm{~s}$ of $12 \mathrm{~s}$ signals of each gesture were segmented and labeled for classification. It was to exclude the transient state between two gestures. Thus, 10s stationary sEMG signal containing 10000 frames were extracted for further processing and analysis. Then, the stationary section has the same segmented methods except the effective segmentation area is in the middle of standard section. Only $12 \mathrm{~s}$ to $18 \mathrm{~s}$ signals of each gesture were segmented and labeled for classification. At last, transient segment part was processed. There is a movement process when the gesture changes from a rest state to a specific action and this process will hold for $1 \mathrm{~s}$ or $2 \mathrm{~s}$ in the earlier part of $12 \mathrm{~s}$ signals. To collected enough transient information during the changes between gestures, We defined the earlier $4 \mathrm{~s}$ of $12 \mathrm{~s}$ signals as the transient segment. Only $8 \mathrm{~s}$ to $12 \mathrm{~s}$ signals of each gesture were segmented for training and testing.

The whole dataset was organized in Matlab format with architecture as described in Table I. The files titled in the format of aaa-ccc.mat and ss-aaabbb-ccc.mat indicated the raw and preprocessing data, respectively, where aaa was the subject ID, BBB was the gesture ID, ccc was the session ID and ss represent the three segmented shapes: standard, transient and stationary. For example, 001-002.mat represent the raw sEMG data captured from subject 1 in the second session, and 02-003-004-005.mat contained the transient sEMG data of gesture 4, captured from subject 3 in the fifth session.

\subsection{Feature Extraction and Classification}

TD features outperformed most feature extraction methods. Moreover, it is low calculation. In this paper, we choose four TD features and an AR model with four coefficients as the evaluation features. In this scheme, each analysis window is equal to $300 \mathrm{~ms}$ (300 samples in $1 \mathrm{kHz}$ sampling) and each incremental window is set to $100 \mathrm{~ms}$. The selected four TD features are: Modified mean absolute value (MAV), Waveform length (WL), Root Mean Square(RMS) and Average amplitude change (AAC) .

For the classification of the sEMG signals, three traditional classification methods were implemented in MATLAB tool: SVM, LDA and KNN. Initially, the SVM classification with Radial Basis Function (RBF) kernel were applied and the gamma of kernel function is set to 0.125 .

\section{$3 \quad$ Experiment setup}

To verify the classification with transient signal segments can acquire similar or superior performance than the other two, three types of experiment and evaluation method as listed below. 
Table 2. a comparison of three segmented shapes with different classification algorithms in inter-session evaluation.

\begin{tabular}{|c|c|c|c|c|c|c|c|c|c|}
\hline \multirow{2}{*}{ Method } & \multicolumn{3}{|c|}{ transient } & \multicolumn{3}{|c|}{ stationary } & \multicolumn{3}{|c|}{ standard } \\
\hline & KNN & LDA & SVM & KNN & LDA & SVM & KNN & LDA & SVM \\
\hline WL & 0.7060 & 0.6698 & 0.6585 & 0.6935 & 0.6882 & 0.7220 & 0.6935 & 0.6882 & 0.7224 \\
\hline RMS & 0.6921 & 0.6771 & 0.6621 & 0.6826 & 0.7075 & 0.7298 & 0.6900 & 0.6942 & 0.7213 \\
\hline MAV & 0.7132 & 0.6985 & 0.6258 & 0.6991 & 0.7103 & 0.6504 & 0.6689 & 0.7116 & 0.6962 \\
\hline $\mathrm{AR} 4$ & 0.6744 & 0.7895 & 0.7732 & 0.6391 & 0.8238 & 0.7943 & 0.6528 & 0.8252 & 0.8074 \\
\hline $\mathrm{AAC}$ & 0.6768 & 0.6875 & 0.6620 & 0.6623 & 0.7164 & 0.7097 & 0.6706 & 0.6953 & 0.7295 \\
\hline
\end{tabular}

${ }^{1}$ Abbreviations: Waveform length(WL), Root mean square(RMS), Modified mean absolute value(MAV), Auto-regressive coefficients(AR4), Average amplitude change(AAC).

Table 3. a comparison of three segmented shapes with different classification algorithms in intersubject evaluation.

\begin{tabular}{|c|c|c|c|c|c|c|c|c|c|}
\hline \multirow{2}{*}{ Method } & \multicolumn{3}{|c|}{ transient } & \multicolumn{3}{|c|}{ stationary } & \multicolumn{3}{|c|}{ standard } \\
\hline & KNN & LDA & SVM & KNN & LDA & SVM & KNN & LDA & SVM \\
\hline WL & 0.5743 & 0.5635 & 0.5554 & 0.5397 & 0.5175 & 0.5774 & 0.5406 & 0.5311 & 0.5565 \\
\hline RMS & 0.6143 & 0.5413 & 0.5946 & 0.5362 & 0.5150 & 0.5746 & 0.5486 & 0.5246 & 0.4981 \\
\hline MAV & 0.5983 & 0.5494 & 0.5524 & 0.5465 & 0.5163 & 0.5812 & 0.5558 & 0.5245 & 0.5185 \\
\hline $\mathrm{AR} 4$ & 0.4865 & 0.5612 & 0.6211 & 0.4751 & 0.5621 & 0.5958 & 0.4808 & 0.5645 & 0.5823 \\
\hline $\mathrm{AAC}$ & 0.5742 & 0.6636 & 0.6358 & 0.5396 & 0.5175 & 0.5689 & 0.5389 & 0.5311 & 0.5102 \\
\hline
\end{tabular}




\subsection{Intra-session strategy}

To evaluate the general classification performance, data within one session was used in this experiment. All subjects are independent, so we choose one subjects data to repeat the procedure. Each session will be divided into training data and testing data. The average classification accuracy across six tests was used to evaluate the performance of the three segmented shapes.

\subsection{Inter-session strategy}

Three sessions collected in the same electrode distribution were used for training and the remaining three sessions collected in three days later for testing. Then, the later three sessions for training and earlier three sessions for testing. Although the training and testing data were collected from the same subject, a large gap will exist between them due to electrode shift, etc. The average classification accuracy across eight tests was used to evaluate the performance of the three segmented shapes.

\subsection{Inter-subject strategy}

A single subjects data was chosen to testing the classifier and the remaining data as the training dataset. In the current case, eight training and testing procedures will be executed, the data of one of eight subjects were used as the testing data in turn, and the remaining data as the training data. The average classification accuracy across eight tests was used to evaluate the performance of the three segmented shapes.

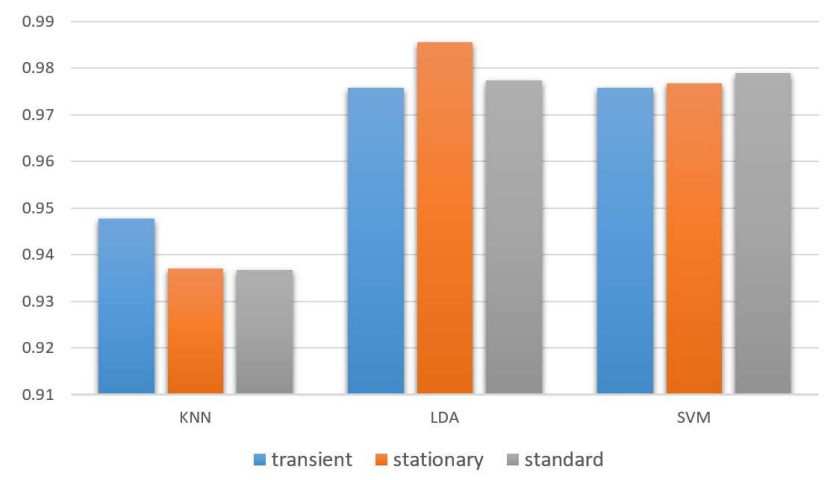

Fig. 5. The classification accuracy between three segemented shapes. 


\section{Yue Zhang et al.}

\section{Results and Discussion}

\section{1 classification accuracy performance}

Three different experiments were implemented in this paper to evaluate the performance of different preprocessing methods. In the intra-session part, a combination of feature extraction and classification methods were applied to the three segmented shapes. The result can be summarized as a histogram in the fig. 5 . The average accuracy is over $90 \%$. LDA can gained more accuracy compared to two other classifications. The transient signal segments between two gestures hidden some important information of muscle contraction.

In the part of inter-session, the experimental results demonstrated that the classification with transient signal segments are similar to the other two, as listed in TABLE II, the highest accuracy that transient segments acquired is $78.95 \%$ with the LDA and AR4. In addition, LDA outperformed the other two classifier in three segmented shapes, it obtains the highest $78.95 \%, 82.38 \%$ and $82.52 \%$, respectively, in the three shapes. AR4 can achieve better characterization of feature vector and get better classification performance with different classifier.

As seen in TABLE III, the classification accuracy of inter-subject can be seen in the table. The accuracy of transient signal segment performed best. It acquired $66.36 \%$ with LDA and AAC, which is $6 \%$ higher than the top accuracy in stationary signal segment and standard signal segment. Besides, the average accuracy of transient is around $55 \%$ to $60 \%$, which illustrate the stability of transient signal segment in inter-subject. Among all the features, AR4 and AAC performed better.

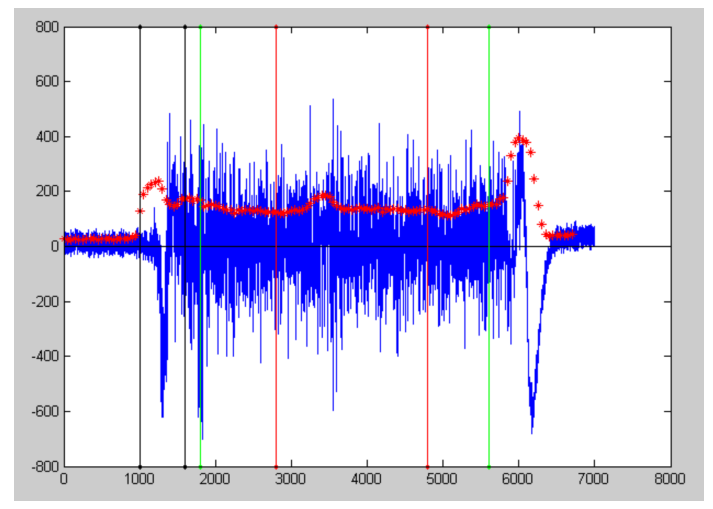

Fig. 6. The three segmented shapes in raw and feature data plot.

\section{2 discussion}

As we can see in the fig. 6 , raw data signals of one gesture was plotted on the figure, blue curve represent the raw signal, the red dotted line represent the RMS 
feature extracted from this signal with the length of analysis window is 100, and shift window is 50. In general, we extract the effective signal between green line, which is standard and steady. Sometimes, real-time performance is required, so signal segment between red lines will be extract as the whole representative of the motion. The signal segment between two black lines in the left is the transient signal which occurs along with hand gestures changes.

In intra-session, the sEMG data is offline, any popular machine learning classifier can extract the hidden model between the training data and theirs labels. However, when external environment changes, model extracted from the stationary signal segments will be expired. Compared to the stationary signal, Transient signal is always related to the changes, which decrease the influences by external factors. Certainly, the classification accuracy with transient signal segments is not very well for daily use, some essential characteristics should be discoverd.

\section{Conclusion}

In this paper, we discuss the transient signal segments acquired when gesture changes. To reveal the useful information hidden in the transient signal, we designed some comparative experiments. Three kinds of signal segments were extracted as the representative sector of hand gestures. Traditional feature extraction methods including WL, AAC, AR4, RMS, MAV and classification methods are combined to evaluate the proposed methods. The results show that transient signal segments can achieve satisfactory result in single session. In addition, transient signal segments can gain more stable representative information between different subjects. It acquired better classification performance in the part of inter-subject. Two referenced segmented shapes, stationary and standard, are all extracted from the steady part of one hand gesture, they can get almost the same performance in three experiments, which illustrate that some representative small stationary signal segment can get better performance in accuracy and calculation cost. In the future, we plan to fit the transient sectors by some regression methods for promoting the robustness of long-term sEMG signals.

\section{References}

1. Jiang N, Dosen S, Muller K, et al. Myoelectric Control of Artificial Limbs-Is There a Need to Change Focus?[J]. IEEE Signal Processing Magazine, 2012, 29(5):152150.

2. Wehner T, Vogt S, Stadler M. Task-specific EMG-characteristics during mental training $[\mathrm{J}]$. Psychological research, 1984, 46(4): 389-401.

3. C. Castellini and P. V. D. Smagt, Surface emg in advanced hand prosthetics, Biol. Cybern., vol. 100, no. 1, pp. 3547, 2009.

4. Cipriani C, Segil J L, Birdwell J A, et al. Dexterous control of a prosthetic hand using fine-wire intramuscular electrodes in targeted extrinsic muscles.[J]. IEEE Transactions on Neural Systems \& Rehabilitation Engineering A Publication of the IEEE Engineering in Medicine \& Biology Society, 2014, 22(4):828-836. 
5. Chu J U, Moon I, Mun M S. A real-time EMG pattern recognition system based on linear-nonlinear feature projection for a multifunction myoelectric hand.[C]// International Conference on Rehabilitation Robotics. IEEE Xplore, 2006:295-298.

6. Englehart, Kevin, and Bernard Hudgins. "A robust, real-time control scheme for multifunction myoelectric control." IEEE transactions on biomedical engineering 50.7 (2003): 848-854.

7. D. Tkach, H. Huang, and T. Kuiken, Study of stability of time-domain features for electromyographic pattern recognition, J. Neuroeng. Rehabil., vol. 7:21, p. 13, 2010.

8. I. S. Jacobs and C. P. Bean, "Fine particles, thin films and exchange anisotropy," in Magnetism, vol. III, G. T. Rado and H. Suhl, Eds. New York: Academic, 1963, pp. $271-350$.

9. L. Hargrove, K. Englehart, and B. Hudgins, A training strategy to reduce classification degradation due to electrode displacements in pattern recognition based myoelectric control, Biomed. Signal Process. Control, vol. 3, pp. 175180, 2008.

10. Phinyomark, Angkoon, Pornchai Phukpattaranont, and Chusak Limsakul. "Feature reduction and selection for EMG signal classification." Expert systems with applications 39.8 (2012): 7420-7431.

11. Ciresan, Dan C., et al. "High-performance neural networks for visual object classification." arXiv preprint arXiv:1102.0183 (2011).

12. Atzori, Manfredo, Matteo Cognolato, and Henning Mller. "Deep learning with convolutional neural networks applied to electromyography data: A resource for the classification of movements for prosthetic hands." Frontiers in neurorobotics 10 (2016): 9.

13. Geng, Weidong, et al. "Gesture recognition by instantaneous surface EMG images." Scientific reports 6 (2016): 36571

14. J. Liu, "Adaptive myoelectric pattern recognition toward improved multifunctional prosthesis control," Med Eng Phys, vol. 37, pp. 424-30, Apr 2015.

15. Chen X, Zhang D, Zhu X. Application of a self-enhancing classification method to electromyography pattern recognition for multifunctional prosthesis control[J]. Journal of neuroengineering and rehabilitation, 2013, 10(1): 44.

16. Y. Fang, X. Y. Zhu, and H. H. Liu, Development of a surface emg acquisition system with novel electrodes configuration and signal representation, in 6th International Conference, ICIRA 2013, pp. 405414.

17. Y. Fang, and H. H. Liu, Robust sEMG Electrodes Configuration for Pattern Recognition based Prosthesis Control, in 2014 International Conference, ICSMC 2014, pp. 22102215 .

18. Atzori, Manfredo, et al. "Electromyography data for non-invasive naturallycontrolled robotic hand prostheses." Scientific data 1.1 (2014): 1-13.

19. Amma, Christoph, et al. "Advancing muscle-computer interfaces with high-density electromyography." Proceedings of the 33rd Annual ACM Conference on Human Factors in Computing Systems. 2015.

20. G. Y., P. Zhou, and G. Li, Toward attenuating the impact of arm positions on electromyography pattern-recognition based motion classification in transradial amputees, J. NeuroEng. Rehab., vol. 9, no. 74,2012,Available:http://www.jneuroengrehab.com/content/9/1/74/abstract. 